\title{
The Rollover Risk in Delta Tricycles: A New Rollover Index and Its Robust Mitigation by Rear Differential Braking
}

\author{
Martín Antonio Rodríguez Licea $\left(\mathbb{D},{ }^{1}\right.$ Edgar Armando Vazquez Rodríguez, ${ }^{2}$ \\ Francisco Javier Perez Pinal $\mathbb{C}^{2}{ }^{2}$ and Juan Prado Olivares ${ }^{2}$ \\ ${ }^{1}$ CONACYT-Instituto Tecnológico de Celaya, Departamento de Ingeniería Electrónica, Guanajuato 38010, Mexico \\ ${ }^{2}$ Instituto Tecnológico de Celaya, Departamento de Ingeniería Electrónica, Guanajuato 38010, Mexico \\ Correspondence should be addressed to Martín Antonio Rodríguez Licea; martin.rodriguez@itcelaya.edu.mx
}

Received 9 April 2018; Revised 9 August 2018; Accepted 27 September 2018; Published 17 October 2018

Academic Editor: Eric Lefevre

Copyright (C) 2018 Martín Antonio Rodríguez Licea et al. This is an open access article distributed under the Creative Commons Attribution License, which permits unrestricted use, distribution, and reproduction in any medium, provided the original work is properly cited.

\begin{abstract}
Although there are efforts to electrify and diversify small vehicles, active safety on motorcycles and tricycles (also known as auto rickshaw, tuk-tuk, mototaxi, etc.) has been relegated until a few years ago. For instance, the electric tricycles (even the combustion ones) marketed today do not have an active safety system that prevents or mitigates the risk of rollover, despite how prone they are to such a situation. The concern for the increase in its marketing is latent and, unfortunately, there are very few related studies. In this article, we present the obtaining and validation of a new rollover index for tricycles demonstrating its effectiveness in predicting and detecting the risk, even statically, by means of a simple quantity. In addition, a controller for the mitigation of the risk of rollover is presented which, by means of a Lyapunov type analysis, it is shown to be robust to changes in parameters, such as the center of gravity height, using a polytopic representation of the system and a differential braking strategy on the rear wheels. Numerical simulations, including video simulation captures, of the operation of the rollover mitigation system using widely recognized commercial software, are also presented. This work can be extended to vehicles with a suspension system or for trikes without autocamber.
\end{abstract}

\section{Introduction}

In recent years, concern about high levels of pollution has increased due to its effects on climate change and the use of fossil fuels on a large-scale is considered one of its main precursors. An alternative widely accepted by governments is to promote the change to electric transportation since generation of electrical energy can come from green or renewable sources that imply a much lower pollution, in addition to their transportation and commercialization is much more efficient [1-3]. The small vehicles technology has not been the exception to such change, and currently researchers are in full development to achieve an efficiency and economy comparable to those in internal combustion vehicles (see [4-8] and many others). For instance, commercialization of electric tricycles as a green substitute for rickshaws, auto rickshaws, tuk-tuks, and even wheelchairs and other devices for differently abled people, is growing day by day (Flyhorse,
Jinhua Jiabao Vehicle Co., Ltd., Fengxian Hao Ran Electric Vehicle Co., Ltd., Jinyi Vehicle Co., Ltd., Chongqing Tengtian Group, and many others). Unfortunately, while for vehicles of four or more wheels there are numerous commercial systems and global research of new strategies of active safety (see [914] and many others), in two and three wheelers (even those of fossil fuel) there is very little research despite the high speeds that can reach and how prone they are to become unstable.

In [15], the authors presented the system model for velocity control and autonomous trajectory control for an electric tricycle vehicle; a PID controller is used and software simulations are presented; however a curvature path is calculated and it is considered that the tricycle can do any trajectory. An interesting comparative study of Fuzzy and Neural controllers is presented in [16]; although in some of these strategies a turn limit is considered, lateral stability is not assured; that is, they are still prone to a rollover. In 
$[17,18]$, the authors presented a model of risk based on traffic and/or GPS information. Although this strategy could take advantage of this information to avoid accidents related to rollover, a priori knowledge is required and lateral stability could only be ensured for a limited set of situations. The authors in [19] presented the most related investigation about lateral stability behaviour in a three wheeled vehicle. In such paper, a static limit on the lateral acceleration is imposed to avoid the tipping of some rear wheel when the center of gravity $(C o G)$ is centered with respect to the rear axle; however, in a real scenario the position of the center of gravity is hard to estimate and time-variant. In addition, the authors present neither a mitigation strategy for the lateral acceleration nor a dynamical validation of the evaluation strategy of the risk.

Although a rollover index (RI) is found in the regulations for 4 and more wheels vehicles ([20]), it is presented as a definition but not as a way to estimate it, much less to mitigate it through an active security system. Many authors have used this definition to generate tractable mathematical models for estimating the RI to mitigate the risk of rollover in 4-wheel vehicles even using multiple actuators (see, for instance, [13, 21-25]). However, the rollover dynamics of a 3-wheel vehicle in delta configuration (a front wheel and two rear wheels) differs considerably from that in a 4 -wheel vehicle and its modelling is not a trivial task; at the moment, there is not in the literature a mathematical modelling that allows the dynamical estimation of the rollover risk in tricycles.

Even more, the mitigation of the rollover in wheeled vehicles is an extremely complicated task, especially in tricycles with a single wheel in front.

In this paper, a dynamical model of the lateral behaviour (rollover dynamics) in a tricycle with a single front steering wheel and in-wheel traction motor and two rear wheels with independent brake is presented; this model can be easily adapted for analogous configurations and is obtained from simple principles and validated by CarSim, a very accepted software in the study of wheeled vehicle designs. Later, a dynamic measure of the rollover risk is presented; such estimation is called rollover index (RI) in allusion to that in 4-wheel vehicles; however the RI estimation for tricycles presented in this paper is novelty.

The mathematical model presented in this paper is suitable to allow the mathematical design of RI mitigation controllers and devices (is a tractable rollover model). The RI presented in this paper is an easily comprehensible quantity within the $[-1,1]$ range, where the sign indicates the side to which the risk is presented and its magnitude indicates the level of risk ( 1 indicates an imminent rollover to the right).

For instance, in this paper, a polytopic representation of the mathematical model is used to design a controller that uses rear differential braking as an actuator and that is robust to parametric uncertainty; this includes the position of the center of gravity, and it is mathematically demonstrated that the strategy can mitigate the risk of rollover as far as the limits of physics allow. Plainly, different control strategies could be used each one with advantages and disadvantages; however, a polytopic representation allow designing a controller that is robust against parameter variation within quantitative

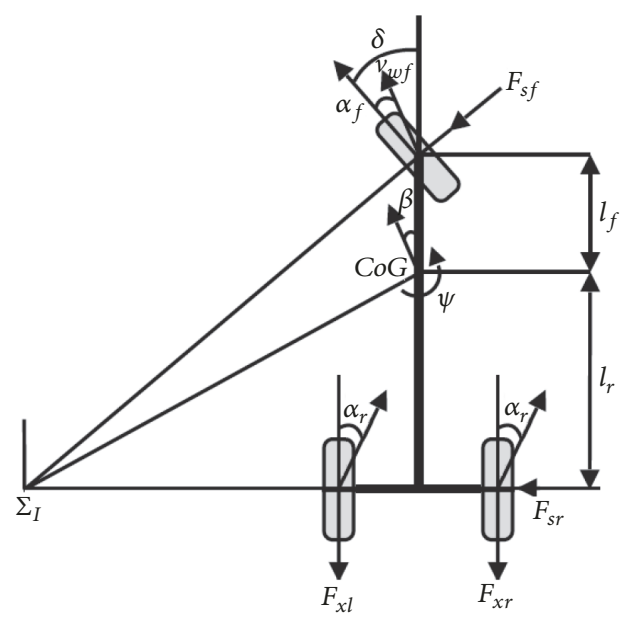

FIGURE 1: Idealization for lateral dynamics of the tricycle.

compact ranges, ensuring that the rollover risk is mitigated; only sensitive parameters can be selected to have a variation, and the gains are obtained offline allowing a fine tuning (regularly P, PD, PI, or PID controllers are used).

Finally, illustrative simulations are presented using the mathematical model generated by CarSim to show that the differential braking controller is capable of mitigating the RI.

Organization of this paper is as follows. In Section 2 the tricycle mathematical model is presented. In Section 3 the RI deduction is exposed and in Section 4 we have validations of the dynamic model behaviour and of the RI ability to predict and detect a rollover. The robust RI mitigation controller is presented in Sections 5 and 6 illustrative simulations. Final discussion and conclusions are presented in Sections 7 and 8, respectively.

\section{Tricycle Model}

In order to obtain the dynamic lateral behaviour of the tricycle, Figure 1 is used. From the point of view of an observer in the origin of an inertial coordinate system $\Sigma_{I}$,

$$
\begin{aligned}
& v_{w f} \sin \left(\delta-\alpha_{f}\right)=l_{f} \dot{\psi}+v_{C o G} \sin (\beta) \\
& v_{w f} \cos \left(\delta-\alpha_{f}\right)=v_{C o G} \cos (\beta)
\end{aligned}
$$

where $C o G$ is the center of gravity, $v_{w f}$ is the front wheel velocity, $\delta$ is the front wheel angle with respect to a longitudinal axis fixed at the tricycle frame (steering angle), $\alpha_{f}$ is the angle difference between the velocity of the front wheel and a longitudinal axis fixed at the center of the front wheel (sideslip angle), $l_{f}$ is the distance from the center of the front wheel to the CoG, $\psi$ is the angle of the longitudinal axis fixed to the frame of the tricycle with respect to an inertial coordinate system, $v_{\mathrm{CoG}}$ is the magnitude of the velocity of the $C o G$, and $\beta$ is the angle difference between the velocity of the $\operatorname{Co} G$ and the longitudinal axis fixed to the frame of the tricycle. Here it is considered a planar translation (over the $X Y$ plane only) without slipping of the wheels. Dividing 1 by 
2 and considering that $v_{\mathrm{CoG}}>0$ and that $\delta$ and $\alpha_{f}$ are small angles $\sin (\beta) \approx \beta, \cos (\beta) \approx \beta$, and $\tan \left(\alpha_{f}\right) \approx \alpha_{f}$,

$$
\alpha_{f}=\delta-\beta-\frac{l_{f} \dot{\psi}}{v_{C o G}}
$$

If the coordinate system $\Sigma_{I}$ is far enough, the rear wheels can be seen as a single one and performing a similar analysis is obtained:

$$
\alpha_{r}=-\beta+\frac{l_{r} \dot{\psi}}{v_{C o G}}
$$

where $\alpha_{r}$ is the angle difference between the velocity of the rear wheels and a longitudinal axis fixed at the center of the front wheel (sideslip angle), and $l_{r}$ is the distance from the center of the rear wheels to the CoG.

On the other hand, the translational dynamics is

$$
\left[\begin{array}{c}
\dot{x}_{i n} \\
\dot{y}_{i n}
\end{array}\right]=v_{C o G}\left[\begin{array}{c}
\cos (\beta+\psi) \\
\sin (\beta+\psi)
\end{array}\right]
$$

Differentiation of (5) turns on

$$
\begin{aligned}
& {\left[\begin{array}{c}
\ddot{x}_{i n} \\
\ddot{y}_{\text {in }}
\end{array}\right]=} v_{C o G}(\dot{\beta}+\dot{\psi})\left[\begin{array}{c}
-\sin (\beta+\psi) \\
\cos (\beta+\psi)
\end{array}\right] \\
&+\dot{v}_{C o G}\left[\begin{array}{l}
\cos (\beta+\psi) \\
\sin (\beta+\psi)
\end{array}\right]
\end{aligned}
$$

A coordinate transformation to a coordinate system fixed in the tricycle frame $\Sigma_{C o G}$ allows obtaining

$$
\left[\begin{array}{c}
\ddot{x} \\
\ddot{y}
\end{array}\right]=v_{C o G}(\dot{\beta}+\dot{\psi})\left[\begin{array}{c}
-\sin (\beta) \\
\cos (\beta)
\end{array}\right]+\dot{v}_{C o G}\left[\begin{array}{c}
\cos (\beta) \\
\sin (\beta)
\end{array}\right]
$$

Newton's second law in the same scenario can be written as

$$
\left[\begin{array}{l}
\ddot{x} \\
\ddot{y}
\end{array}\right]=\frac{1}{m}\left[\begin{array}{c}
\Sigma F_{x} \\
\Sigma F_{y}
\end{array}\right]
$$

where $m$ is the vehicle total mass, $\Sigma F_{x}$ is the total longitudinal force, and $\Sigma F_{y}$ is the total lateral force. Considering that $v_{\mathrm{CoG}}$ is a varying parameter with a nominal value, then $\dot{v}_{C o G} \approx 0$. That is, for now the speed of the CoG is considered constant and in the following sections a Linear Parameter Varying (LPV) system is built in order to obtain a robust design controller for quantitative limits of $v_{\mathrm{CoG}}$. From (7) and (8), $\dot{\beta}$ can be written as

$$
\dot{\beta}=\frac{\cos (\beta)}{m v_{C o G}} \Sigma F_{y}-\frac{\sin (\beta)}{m v_{C o G}} \Sigma F_{x}-\dot{\psi}
$$

Considering that the rolling resistance, aerodynamic drag, and other effects are compensated by the traction of the inwheel front motor to maintain a speed, the longitudinal force in the front wheel balance is zero (in this case the tricycle has only traction). $\Sigma F_{x}=F_{x l}+F_{x r}$, where $F_{x l}$ is the braking force in the left rear wheel and $F_{x r}$ is the braking force in the right rear wheel. From Figure 1 the lateral force balance can be calculated as $\Sigma F_{y}=F_{y f}+F_{y f}$, where $F_{y f}$ is the lateral force in the center of the front wheel, $F_{y r}$ is the force in the axis of the rear wheels, and

$$
\begin{aligned}
& F_{y f}=F_{s f} \cos (\delta) \\
& F_{y r}=F_{s r}
\end{aligned}
$$

where $F_{s f}$ is the front lateral force component of the front lateral force with respect to $\Sigma_{C o G}$ and $F_{s r}$ is the rear lateral force component of the rear lateral force with respect to $\Sigma_{C o G}$ (there is no rear steering). The lateral forces can be approximated by nonlinear functions of the tire sideslip angle for a wide region of lateral force $([26,27])$. In this paper, the lateral forces are approximated by linear functions since the main objective is to conservatively predict a rollover risk; this is, the nonlinear approaches exhibit a saturation behaviour while the linear ones predict larger lateral forces and, for instance, larger rollover risks as developed in the following sections. Using (3) and (4), the lateral forces can be written as

$$
\begin{aligned}
F_{y f} & =F_{s f} \cos (\delta)=c_{f} \alpha_{f} \cos (\delta) \\
& =c_{f}\left(\delta-\beta-\frac{l_{f} \dot{\psi}}{v_{C o G}}\right) \cos (\delta) \\
& \approx c_{f}\left(\delta-\beta-\frac{l_{f} \dot{\psi}}{v_{C o G}}\right) \\
F_{y r} & =F_{s r}=c_{r} \alpha_{r} \approx c_{r}\left(-\beta+\frac{l_{r} \dot{\psi}}{v_{C o G}}\right)
\end{aligned}
$$

From (9), (13), and the small $\beta$ approximation, $\dot{\beta}$ can be rewritten as

$$
\dot{\beta}=-\frac{c_{f}+c_{r}}{m v_{C o G}} \beta+\left[\frac{c_{r} l_{r}-c_{f} l_{f}}{m v_{\mathrm{CoG}}^{2}}-1\right] \dot{\psi}+\frac{c_{f}}{m v_{C o G}} \delta
$$

The previous equation depends on $\dot{\psi}$; the dynamics of the yaw rate can be obtained performing a torque balance (Figure 1):

$$
J \ddot{\psi}=l_{r} F_{y r}+l_{f} F_{y f}+b_{l} F_{x l}-b_{r} F_{x \mathrm{r}}
$$

where $J$ is the yaw moment of inertia, $b_{l}$ is the distance from the left rear wheel center to the frame longitudinal axis, and $b_{r}$ is the distance from the right rear wheel center to the frame longitudinal axis. Note that these last parameters are considered different since the driver of the tricycle can be inclined to a side, moving CoG instead of being in the center. Substituting the forces the dynamical behaviour of the yaw rate is

$$
\begin{aligned}
\ddot{\psi}= & \frac{l_{r} c_{r}-l_{f} c_{f}}{J} \beta+\frac{l_{r}^{2} c_{r}-l_{f}^{2} c_{f}}{J v_{C o G}} \dot{\psi}+\frac{l_{f} c_{f}}{J} \delta+\frac{b_{l}}{J} F_{x l} \\
& -\frac{b_{r}}{J} F_{x r}
\end{aligned}
$$




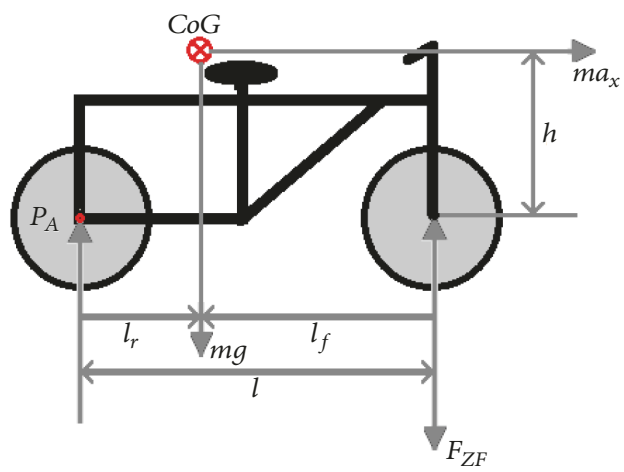

FIgURE 2: Idealization for front-to-rear, vertical dynamics of the tricycle.

\section{Rollover Detection}

A rollover condition can be obtained from a wheel vertical forces unbalance $[28,29]$. However, for the case of the tricycle, the rollover index found in the literature is no longer valid since $C o G$ is always laterally centered between left and right rear wheels (that is, $b_{r}=b_{l}$ ). In a tricycle, the longitudinal and lateral positions of $C o G$ have an important effect on the rollover risk. The rollover index is defined as the normalized difference between the left and right vertical forces; that is, when they are equal the risk is zero, if left vertical forces are zero the risk is one, and if right vertical forces are zero the risk is minus one. The closer the absolute value of the rollover index to the unit, the higher the risk of tipping, and the sign indicates the side to which the risk is presented. In this paper, the tipping of the front wheel is not considered; that is, the front electric motor does not have enough torque, and there is no front wheel brake to induce a front/full-rear tipping. Under such considerations the rollover index is defined as

$$
R I=\frac{F_{Z R L}-F_{Z R R}}{F_{Z R L}+F_{Z R R}}
$$

where $F_{Z R L}$ and $F_{Z R R L}$ are the vertical forces in the tireground contact point for the left and right wheels, respectively. Using Figure 2 and performing a front-to-rear torque balance with respect to the point $P_{A}$ one has

$$
F_{Z F}=\frac{l_{r} m g}{l}+\frac{h m a_{x}}{l}
$$

where $F_{Z F}$ is the vertical force for the front wheel in the tireground contact point, $l$ is the distance between front and rear axles, $g$ is the gravitational acceleration, $h$ is the height of the $\operatorname{CoG}$, and $a_{x}$ is the longitudinal acceleration. For now, the speed of $C o G$ is considered constant and in the following sections a Linear Parameter Varying (LPV) system is built in order to obtain a robust design controller for quantitative limits of $v_{\mathrm{Co} G}$; under this consideration $a_{x} \approx 0$ :

$$
F_{Z F}=\frac{l_{r} m g}{l}
$$

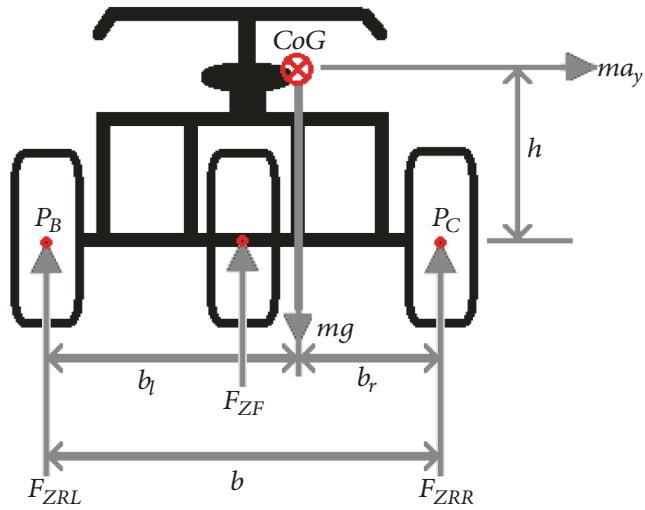

FIgURE 3: Idealization for right-to-left, vertical dynamics of the tricycle.

On the other hand, using Figure 3 and performing a right-toleft torque balance with respect to the point $P_{B}$ one has

$$
-b F_{Z R R}-\frac{b}{2} F_{Z F}+b_{l} m g+h m a_{y}=0
$$

where $F_{Z R R}$ is the vertical force for the rear right wheel in the tire-ground contact point, $b=b_{r}+b_{l}$ is the width of the rear axle, $b_{r}$ is the distance from the rear right wheel to CoG, $b_{l}$ is the distance from the rear left wheel to the $\operatorname{CoG}, h$ is the height of $C o G$, and $a_{y}$ is the lateral acceleration of the CoG. Performing a right-to-left torque balance with respect to the point $P_{C}$ one has

$$
b F_{Z R L}+\frac{b}{2} F_{Z F}-b_{r} m g+h m a_{y}=0
$$

Substituting (19) into (20) and (21) turns on

$$
\begin{aligned}
& F_{Z R R}=-\frac{l_{r} m g}{2 l}+\frac{b_{l} m g}{b}+\frac{m h a_{y}}{b} \\
& F_{Z R L}=-\frac{l_{r} m g}{2 l}+\frac{b_{r} m g}{b}-\frac{m h a_{y}}{b}
\end{aligned}
$$

Using the above equations on (17),

$$
R I=l \frac{b_{r}-b_{l}}{b l_{f}}-\frac{2 h l}{b g l_{f}} a_{y}
$$

From the previous equation, it is easily seen that the RI can be estimated from a simple measure (accelerometer); CoG position parameters can be considered initially constants with their nominal values. In the following, a robust controller is designed, and a stability analysis is performed; that is, the RI for delta tricycles can be experimentally estimated by (24) and the following analysis is performed to demonstrate that a differential braking controller can mitigate the rollover risk in delta tricycles.

Note that there is a direct relationship of the rollover index with the position of the $C o G$; for instance, if $C o G$ is not laterally centered $\left(b_{r} \neq b_{l}\right)$ there is a constant contribution to the risk. Note that if $C o G$ is at the same height as the axles 
TABLE 1: Parameters used for the tricycle model validation.

\begin{tabular}{lcc}
\hline Parameter & Value & Units \\
\hline$b_{l}$ & 0.525 & $\mathrm{~m}$ \\
$b_{r}$ & 0.525 & $\mathrm{~m}$ \\
$c_{f}$ & 120,000 & $\mathrm{~N} / \mathrm{rad}$ \\
$c_{r}$ & 155,000 & $\mathrm{~N} / \mathrm{rad}$ \\
$g$ & 9.8 & $\mathrm{~m} / \mathrm{s}^{2}$ \\
$h$ & 0.54 & $\mathrm{~m}$ \\
$J$ & 1111 & $\mathrm{kgm}^{2}$ \\
$l_{f}$ & 1.103 & $\mathrm{~m}$ \\
$l_{r}$ & 0.922 & $\mathrm{~m}$ \\
$m$ & 747 & $\mathrm{~kg}$ \\
$v_{C o G}$ & 13.9 & $\mathrm{~m} / \mathrm{s}$ \\
\hline
\end{tabular}

height and it is centered laterally, the risk is null; however, this is fiscally hard to accomplish at every time and a control system must be implemented in order to mitigate the risk of rollover. Note also that the closer the center of gravity of the front tire is, the more easily the risk of rollover is triggered by any value of $a_{y}$ different from zero; therefore, in the case of the tricycle with front wheel drive and rear braking, it is recommended that the center of gravity be placed as close to the rear axle as possible (placing the batteries in the back).

The lateral acceleration can be estimated from the next lateral force relation and (12)-(13) as follows:

$$
\begin{aligned}
a_{y} & \approx \frac{F_{y f}+F_{y r}}{m} \\
& \approx-\frac{c_{f}+c_{r}}{m} \beta+\frac{l_{r} c_{r}-l_{f} c_{f}}{m v_{C o G}} \dot{\psi}+\frac{c_{f}}{m} \delta
\end{aligned}
$$

Finally the RI, for controller design purposes, is calculated from the dynamic variables of the vehicle state space model:

$$
\begin{aligned}
R I= & l \frac{b_{r}-b_{l}}{b l_{f}} \\
& -\frac{2 h l}{b g l_{f}}\left(\frac{c_{f}+c_{r}}{m} \beta+\frac{l_{f} c_{f}-l_{r} c_{r}}{m v_{\mathrm{Co} G}} \dot{\psi}-\frac{c_{f}}{m} \delta\right)
\end{aligned}
$$

\section{Model Validation}

In this section, the validation of the mathematical model of the vehicle and the effectiveness of the $R I$ to predict a rollover is presented by using a widely accepted software named CarSim. This validation is performed by comparing the data provided by CarSim and that is generated by the mathematical model integration in Matlab for representative manoeuvres (double lane changes at constant speeds). A tipping is considered when the vertical force in one of the rear wheels is zero, and in this paper such situation is considered an imminent rollover. The parameters of Table 1 are used in the model and in CarSim; however, CarSim uses lateral force profiles instead of linear approximations and the cornering stiffness is obtained from such profiles for front and rear

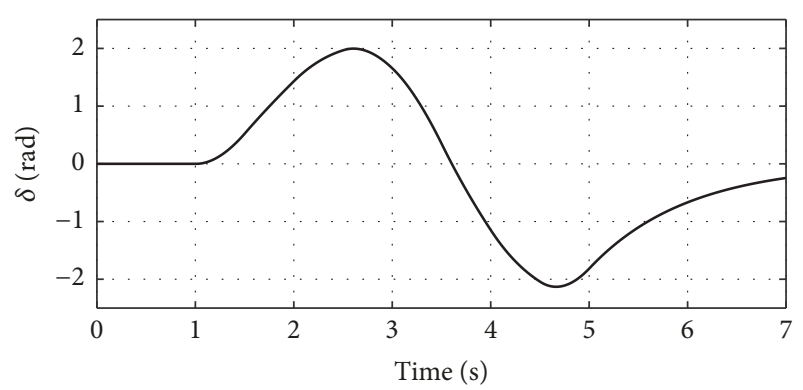

Figure 4: Double lane change manoeuvre.

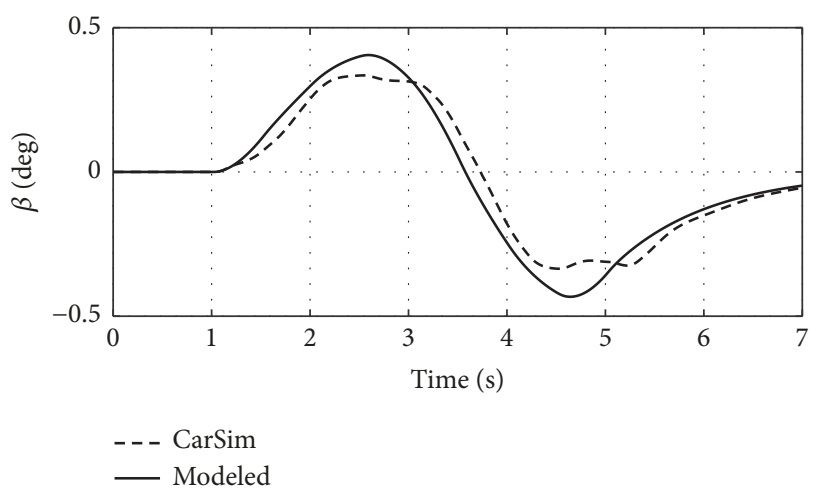

Figure 5: Comparison of the sideslip for CarSim versus the proposed model of the tricycle.

wheels. The front steering has a $1: 19$ reduction and some units conversion is necessary; the friction coefficient is set to 0.85. CarSim uses a suspension system but our tricycle does not have one; the spring rates for the suspension system were set to a very high value in CarSim in order to simulate without such system.

4.1. Tricycle Model Validation. For this validation, the manoeuvre is selected to almost lift a rear wheel (almost a rollover). This is of interest for this study, to maintain the absolute value of $R I$, in lower values instead of making a recovery of the tipping. In Figure 4 the $\delta$ angle is presented, which represents a double lane change. All the simulations on this section were performed at $v_{C o G}=14 \mathrm{~m} / \mathrm{s}$. In Figure 5 a graphic comparison of the sideslip angle $(\beta)$ is shown; it can be noticed that the proposed model performs similar dynamics to CarSim except for nonlinearities. It can be considered a sufficiently precise approximation since the model allows obtaining conservative dynamics. On the other hand, the comparison between CarSim and the presented dynamic model for the dynamics of the yaw rate (Figure 6) and the lateral acceleration (Figure 7 ) is very accurate.

4.2. Rollover Index Validation. The validation of the RI is performed by comparing the vertical forces in the rear wheels provided by CarSim with the RI value. That is, a low value of vertical force in a wheel indicates that this wheel is about to lose contact with the ground and has a high risk of tipping; in this situation the RI absolute value must be almost the unit 


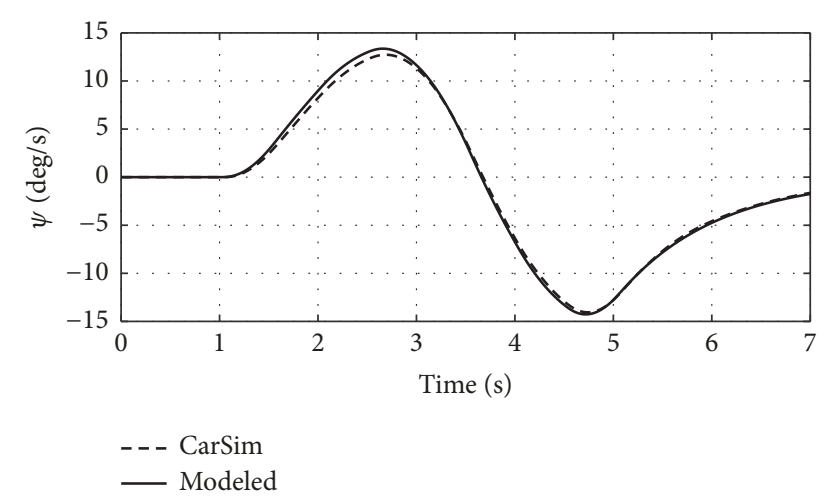

Figure 6: Comparison of the yawrate for CarSim versus the proposed model of the tricycle.

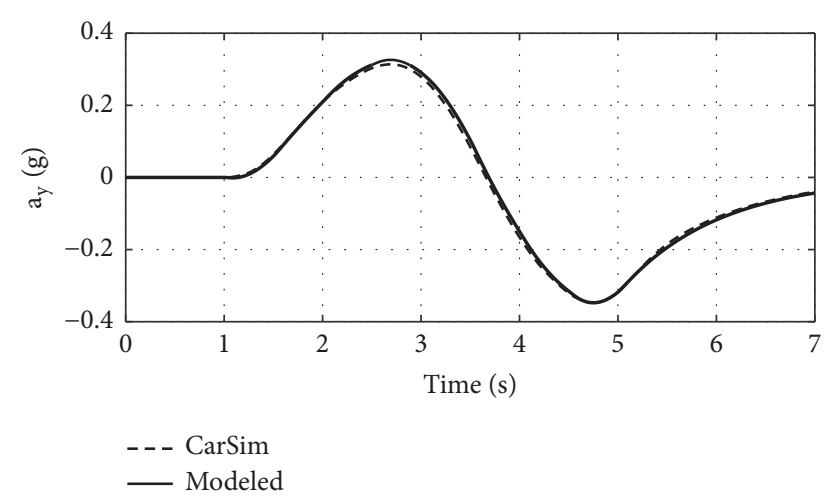

FIGURE 7: Comparison of the lateral acceleration for CarSim versus the proposed model of the tricycle.

and the sign indicates if the risk is to the right $(R I>0)$ or to the left $(R I<0)$.

First, the manoeuvre of Figure 4 is used. In Figure 8 the vertical forces in the rear wheels are shown; these forces are normalized (divided by $m g$ ) for easy comparison with the RI. Also, the RI value is shown. It can be easily noticed that a low vertical force in the left rear wheel force implies a high vertical force in the right rear wheel (a left turn is performed) in $t=2.7 \mathrm{~s}$, such that the RI measures about 0.6 indicating that there is no risk of rollover during a left turn. At $t=4.8$ $s$ the RI is calculated about -0.7 indicating that there is no risk of rollover during a right turn. In Figure 9 an illustrative (pictorial) capture of the CarSim's simulation at $t=2.7 \mathrm{~s}$ $(R I=0.6)$ is shown; the yellow vertical arrows indicate the magnitude of the vertical force for each wheel; note that the rear left vertical force is lesser than the rear right vertical force confirming that there is no risk of rollover during a left turn as the $R I$ value indicates.

Secondly, the manoeuvre of Figure 4 is multiplied by 1.5 $(150 \%)$ and used as the steering input in order to simulate a risky manoeuvre. In Figure 10 the normalized vertical forces in the rear wheels are shown and also the RI value is shown. It can be easily noticed that a very low vertical force in the left rear wheel force implies a very high vertical force in the right rear wheel (a left turn is performed) in $t=2.7 \mathrm{~s}$, such that the RI measures about 0.9 indicating that there

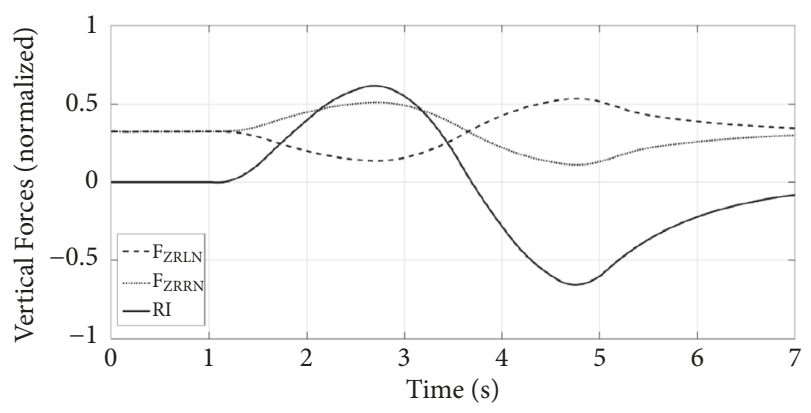

FIGURE 8: Comparison of the RI versus the actual vertical forces in the rear wheels (normalized) during a normal (no rollover risk) manoeuvre.

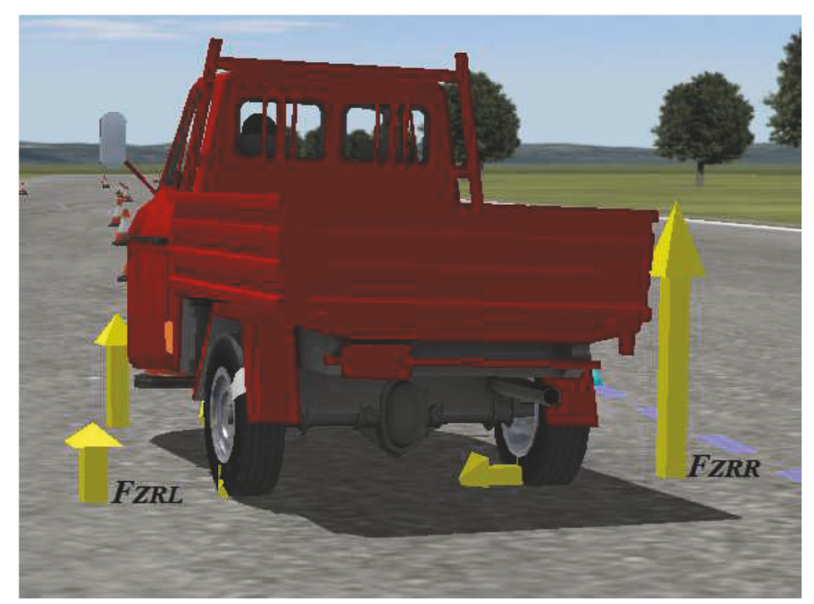

FIGURE 9: Illustrative simulation of the actual vertical forces in the rear wheels at $t=2.7 \mathrm{~s}$.

is a high risk of rollover during a left turn. In Figure 11 an illustrative (pictorial) capture of CarSim's simulation at $t=$ $2.7 s$ confirming a high rollover risk is shown (the rear left vertical force is almost zero).

Finally, the manoeuvre of Figure 4 is multiplied by 1.9 (190\%) and used as the steering input in order to simulate a rollover manoeuvre. In Figure 12 the normalized vertical forces in the rear wheels are shown and also the RI value is shown. It can be easily noticed that a zero vertical force in the left rear wheel force indicates an imminent tipping from $t=$ $1.2 \mathrm{~s}$, such that the RI measures more than 1 , indicating that there is an imminent rollover during a left turn. In Figure 13 an illustrative (pictorial) capture of the CarSim's simulation is shown confirming a left tipping; after $t=2.5 \mathrm{~s}$ the vehicle completely rolls over.

4.3. Discussion. From the previous validation, it is important to highlight the accuracy of the mathematical model and the RI to detect a rollover risk. Since the RI depends on lateral acceleration, it can be measured easily in a real application considering an initial $C o G$ position (nominal parameters), and a robust controller design allows ensuring stability even against abrupt variations of the parameters as long as the values of the parameters are within the compact 


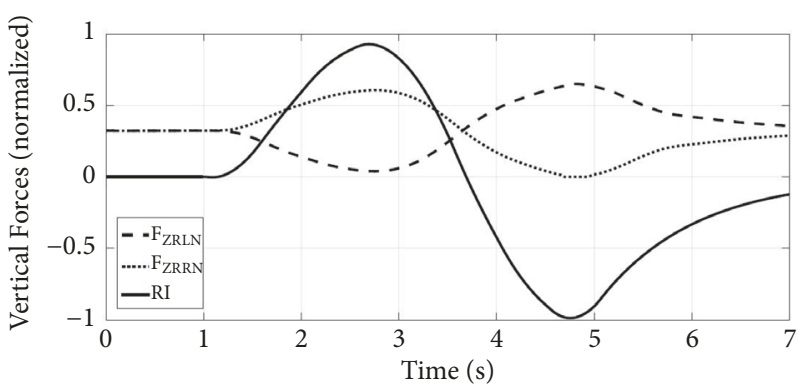

Figure 10: Comparison of the RI versus the actual vertical forces in the rear wheels (normalized) with $150 \%$ of manoeuvre amplitude increment.

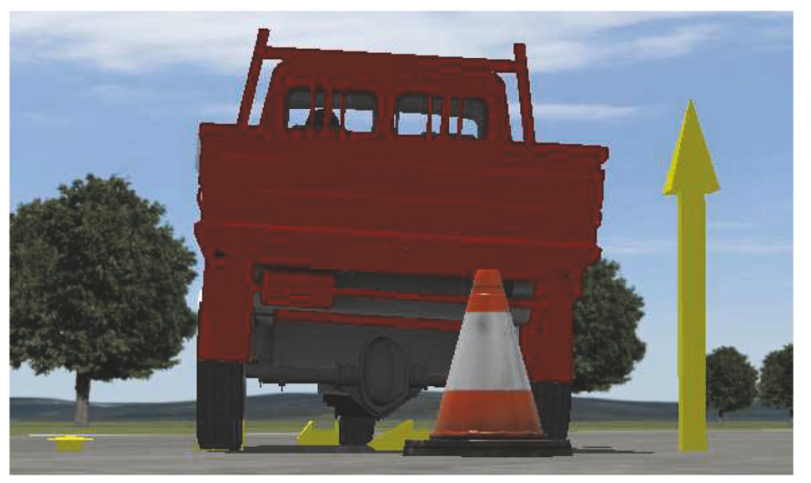

FiguRE 11: Illustrative simulation of the actual vertical forces in the rear wheels at $t=2.7 \mathrm{~s}$ with $150 \%$ of manoeuvre amplitude increment.

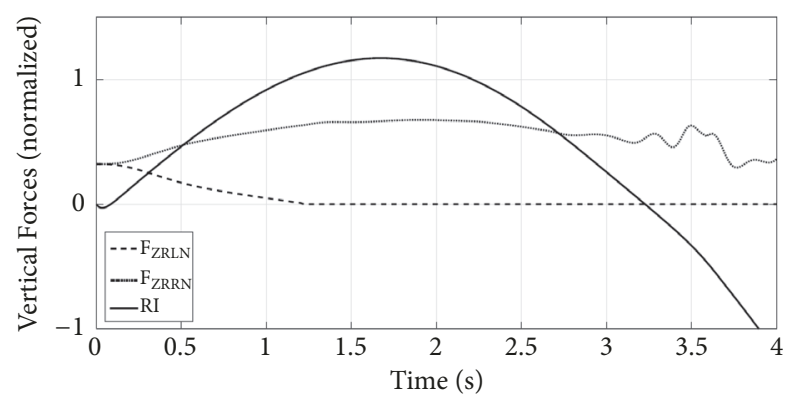

FIGURE 12: Comparison of the RI versus the actual vertical forces in the rear wheels (normalized) with $190 \%$ of manoeuvre amplitude increment.

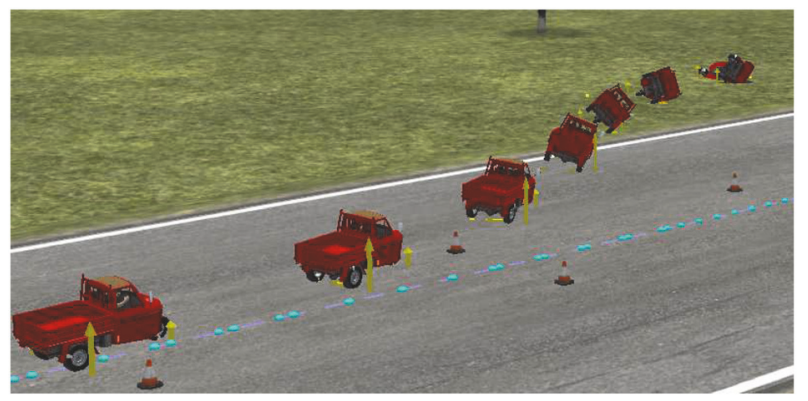

FIGURE 13: Illustrative simulation of the vehicle dynamics at $t=0.8$, $t=1.3, t=1.8, t=2.3 t=2.8, t=3.3$, and $t=3.8 s$ (from left to right) with $190 \%$ of manoeuvre amplitude increment. design ranges. In the following a robust controller design is presented.

\section{Robust Controller Design for the RI Mitigation}

From the first term of the right side of (27), it can be seen that the geometric position of the $C o G$ has a very high impact on the rollover risk. Indeed, if $C o G$ is located near the front of the tricycle or beyond the rear axle, an imminent rollover is calculated even statically. For this reason, a differential braking controller can only mitigate the rollover risk. Despite the above physical limitation, the RI is used to design an output feedback instead of a state feedback as follows.

Conforming/rewriting the complete math model of the system from (16), (14), and (27) one has

$$
\begin{aligned}
& \dot{x}=A_{o l} x+B_{u} u+B_{\delta} \delta \\
& y=C x+D \delta+E_{o l}
\end{aligned}
$$

where

$$
\begin{aligned}
A_{o l} & =\left[\begin{array}{cc}
-\frac{c_{f}+c_{r}}{m v_{C o G}} & \frac{c_{r} l_{r}-c_{f} l_{f}}{m v_{C o G}^{2}}-1 \\
\frac{c_{r} l_{r}-c_{f} l_{f}}{J} & -\frac{c_{r} l_{r}^{2}+c_{f} l_{f}^{2}}{J v_{C o G}}
\end{array}\right], \\
B_{u} & =\left[\begin{array}{ll}
0 & 0 \\
\frac{b_{l}}{J}-\frac{b_{r}}{J}
\end{array}\right], \\
B_{\delta} & =\left[\begin{array}{l}
\frac{c_{f}}{m v_{C o G}} \\
\frac{l_{f} c_{f}}{J}
\end{array}\right], \\
C & =\left[\begin{array}{l}
\frac{2 h l\left(c_{f}+c_{r}\right)}{b g l_{f}} \\
\frac{2 h l\left(l_{f} c_{f}-l_{r} c_{r}\right)}{b g l_{f} m v_{C o G}}
\end{array}\right], \\
D & =-\frac{c_{f}}{m}, \\
E_{o l} & =\frac{l\left(b_{r}-b_{l}\right)}{l_{f} b},
\end{aligned}
$$

$x=[\beta, \dot{\psi}]^{T}, y=R I$, and $u=\left[F_{x l}, F_{x r}\right]^{T}$. Consider the differential braking control law $B_{u} u=[0,-k y]^{T}$ as follows:

$$
\epsilon \frac{b_{l}}{J} F_{x l}-(1-\epsilon) \frac{b_{r}}{J} F_{x r}=-k y
$$

where $k>0$ is a controller gain, $F_{x l} \geq 0, F_{x r} \geq 0$, and $\epsilon \epsilon$ $\{0,1\}$ is zero when a rollover risk to the left is detected and one when to the right. That is, when a rollover risk is detected one should brake only with the exterior wheel. 
Under the above control law, system (28)-(29) is reduced

where to

$$
\dot{x}=A x+B \delta+E
$$

$$
\begin{aligned}
A & =\left[\begin{array}{cc}
-\frac{c_{f}+c_{r}}{m v_{C o G}} & \frac{c_{r} l_{r}-c_{f} l_{f}}{m v_{C o G}^{2}}-1 \\
\frac{c_{r} l_{r}-c_{f} l_{f}}{J}-k \frac{2 h l\left(c_{f}+c_{r}\right)}{b g l_{f}} & -\frac{c_{r} l_{r}^{2}+c_{f} l_{f}^{2}}{J v_{C o G}}-k \frac{2 h l\left(l_{f} c_{f}-l_{r} c_{r}\right)}{b g l_{f} m v_{C o G}}
\end{array}\right], \\
B & =\left[\begin{array}{c}
\frac{c_{f}}{m v_{C o G}} \\
\frac{c_{f} l_{f}}{J}+k \frac{c_{f}}{m}
\end{array}\right], \\
E & =-k \frac{l\left(b_{r}-b_{l}\right)}{l_{f} b},
\end{aligned}
$$

In the following, parametric uncertainty is considered; that is, every value of all parameter in system (32) is considered unknown but within a known range and with a nominal value usually in the middle of the range. For instance, $c_{f}$ has a nominal value of 120,000 and with a variation ratio of $\pm 10 \%$ the range of variation is $\left[c_{f}, \overline{c_{f}}\right]=[108,000 ; 132,000]$ and the minimum and maximum values of the range are denoted by an underline and overline, respectively. System (32) with parametric uncertainty is

$$
\dot{x}=A(t) x+B(t) \delta+E(t)
$$

System (34) can be written as a polytopic, simplex system [30] (in the following named polytopic system for simplicity) using the maximum and minimum of the matrices entries $\left(2^{n}\right.$ entries decomposition for each matrix) in order to establish stability conditions and to determine a suitable controller gain:

$$
\begin{aligned}
\dot{x}= & \theta_{1} A_{1} x+\theta_{2} A_{2} x+\cdots+\theta_{16} A_{16} x+\vartheta_{1} B_{1} \delta+\cdots \\
& +\vartheta_{4} B_{4} \delta+\sigma_{1} E_{1}+\sigma_{2} E_{2}
\end{aligned}
$$

where $\theta_{1}+\theta_{2}+\cdots+\theta_{16}=1, \vartheta_{1}+\cdots+\vartheta_{4}=1, \sigma_{1}+\sigma_{2}=1$, $\theta_{1}, \theta_{2}, \ldots, \theta_{16} \geq 0, \vartheta_{1}, \ldots, \vartheta_{4} \geq 0, \sigma_{1}, \sigma_{2} \geq 0$ (simplices), and

$$
\begin{aligned}
& A_{1}=\left[\begin{array}{ll}
\underline{a}_{1,1} & \underline{a}_{1,2} \\
\underline{a}_{2,1} & \underline{a}_{2,2}
\end{array}\right], \\
& A_{2}=\left[\begin{array}{ll}
\bar{a}_{1,1} & \underline{a}_{1,2} \\
\underline{a}_{2,1} & \underline{a}_{2,2}
\end{array}\right], \\
& A_{3}=\left[\begin{array}{ll}
\underline{a}_{1,1} & \bar{a}_{1,2} \\
\underline{a}_{2,1} & \underline{a}_{2,2}
\end{array}\right],
\end{aligned}
$$

$$
\begin{gathered}
A_{16}=\left[\begin{array}{ll}
\bar{a}_{1,1} & \bar{a}_{1,2} \\
\bar{a}_{2,1} & \bar{a}_{2,2}
\end{array}\right], \\
B_{1}=\left[\begin{array}{l}
\underline{b}_{1} \\
\underline{b}_{2}
\end{array}\right], \\
B_{2}=\left[\begin{array}{l}
\bar{b}_{1} \\
\underline{b}_{2}
\end{array}\right], \\
B_{3}=\left[\begin{array}{l}
\underline{b}_{1} \\
\bar{b}_{2}
\end{array}\right], \\
B_{4}=\left[\begin{array}{l}
\bar{b}_{1} \\
\bar{b}_{2}
\end{array}\right] \\
E_{1}=\underline{E}, \\
E_{2}=\bar{E} .
\end{gathered}
$$

Here, the convex addition property of convex functions is used [30]. From system (35), when the simplices are $\theta_{1}=1$, $\vartheta_{1}=1$, and $\sigma_{1}=1$ the system is known as a vertex of (35); $\theta_{2}=1, \vartheta_{1}=1$, and $\sigma_{1}=1$ are other vertex, successively for all the combinations such that $2^{7}$ vertexes can be found.

In the following, stability conditions of the polytopic system (35) are obtained using the results from [31]: the quadratic stability of system (35) is ensured if it is quadratically stable for all vertexes with a Common Lyapunov Function (CLF). Consider the CLF candidate $\mathrm{V}=x^{T} P x$, where $P$ is a symmetric positive definite matrix, the time derivative of $V$ along the trajectories of the $i$ vertex is

$$
\dot{V}=x^{T}\left(P A_{i}+A_{i}^{T} P\right) x+2\left(B_{i}^{T} \delta+E_{i}^{T}\right) P x
$$


such that if $\dot{V} \leq 0$ for all (i) the quadratic stability is ensured. Consider that $\delta(t) \leq \delta_{\max }$ since the front wheel angle is limited to $\pm \pi / 4$; one should prove that

$$
\begin{array}{r}
x^{T}\left(P A_{i}+A_{i}^{T} P\right) x+2\left(B_{i}^{T} \delta_{\text {max }}+E_{i}^{T}\right) P x \leq \\
x^{T}\left(P A_{i}+A_{i}^{T} P\right) x+\left\|2\left(B_{i}^{T} \delta_{\text {max }}+E_{i}^{T}\right) P\right\|_{2}\|x\|_{2} \leq \\
x^{T}\left(P A_{i}+A_{i}^{T} P+\gamma I\right) x \leq 0
\end{array}
$$

where $I$ is the identity matrix, and $\gamma>0$ is considered a stability margin that depends on the magnitude of the perturbations ( $\delta$ and the uncentering of the $C o G$ decentering/bias). In order to get $P$ and $k$ that maximize the stability margin $\gamma_{\max }$ for all $i$, the following linear programming algorithm is used.

\section{Algorithm 1.}

(1) Set $\gamma_{\max }=0$ and $k=0$.

(2) If $P$ has not be found yet, look for a symmetric, common $P>0$ that solves $P A_{i}+A_{i}^{T} P \preceq 0$.

(3) If a $P$ matrix is found, calculate the maximum stability margin $\gamma_{i}$ for every vertex from $P A_{i}+A_{i}^{T} P+\gamma_{i} I \preceq 0$; else, goto step (6).

(4) Calculate the maximum stability margin $\gamma_{i, \text { max }}$ from the previous step.

(5) If $\gamma_{i, \text { max }}>\gamma_{\text {max }}$ then $\gamma_{\text {max }}=\gamma_{i, \text { max }}$.

(6) Increase $k$ with a small $\Delta_{k}$ value.

(7) If $k \leq k_{\text {max }}$ then goto Step (2).

(8) End.

Here, $>0$ means a positive definite matrix and $\preceq 0$ means a negative semidefinite matrix (in the left side). Step (3) implies solving for $P A_{i}+A_{i}^{T} P+\gamma_{i} I \preceq 0$; this is equivalent to selecting the maximum eigenvalue of $P A_{i}+A_{i}^{T} P$. Note that Step (2) solution is not always guaranteed, depending on the ranges of parametric variation; if a solution can not be found, the parametric ranges should be reduced. $k_{\max }$ is selected accordingly to the maximum braking force, since it is approximately limited to $m g / 2$ in the best scenario (with $l_{r}=0$ and braking with the interior wheel). If a stability margin greater than zero is found, then with some $k>0$ there exists a matrix $P$ suitable to construct a CLF such that the quadratic stability of the polytopic system is ensured.

Moreover, it can easily be seen that the closed loop system allows increasing the stability margin and, therefore, the magnitude of the perturbations; that is, the controller helps to mitigate the RI when the driver performs a turn or when the CoG is not laterally centered. As expected, if the perturbation is of a very high magnitude the rollover is imminent; for instance, if the CoG is laterally beyond the rear axle, it is impossible to avoid the rollover with differential braking.

It is worth mentioning that some of the nominal parameters needed to calculate the control law (31) are not easily measurable, for instance, $b_{l}, b_{r}, l_{f}$, and $h$. However, states/parameters observer can be once established to determine the values nominal and variation ranges; since the controller is robust against bounded parameter changes, it is not necessary an exact estimation of those values.

Finally, it can be concluded that the control law (31) stabilizes system (28)-(29) if the Algorithm 1 can be solved for $\gamma_{\max }>0$ with some $k$, for bounded variation of the parameters.

\section{Simulations}

In this section, an example of the controller design and simulation results is provided. It is worth mentioning that the CarSim model is used in Simulink to close the loop and it uses a nonlinear numeric model to simulate the tricycle dynamics.

Consider a tricycle with the nominal parameters of Table 1 with relevant parametric variation ranges as follows: $b_{l} \in[0.4725 ; 0.5775] m, b_{r} \in[0.4725 ; 0.5775] m, c_{f} \in$ $[115,000 ; 125,000] \mathrm{N} / \mathrm{rad}, c_{r} \in[150,000 ; 160,000] \mathrm{N} / \mathrm{rad}$, $h \in[0.495 ; 0.605] m, l_{f} \in[0.9927 ; 1.2133] m$, and $v_{C o G} \epsilon$ $[1 ; 15] \mathrm{m} / \mathrm{s}$. Note that all the parameters can be considered time-varying if desired. For simulation purposes, hydraulic actuators are used since CarSim provides an input variable consisting of a braking pressure for each rear wheel $\left(P_{b j}\right.$ where $j$ is left/right). The model of the actuator is selected as linear $\left(F x j=380 P_{b j}\right)$ such that $k_{\text {max }}=(m g) /(2 * 380)=9.63$ but the dynamics from the braking process are calculated and included inherently by CarSim. Under the above design one has

$$
\begin{aligned}
\underline{a}_{1,1}= & -\frac{\bar{c}_{f}+\bar{c}_{r}}{m \underline{v}_{C o G}}=-381.5261, \\
\bar{a}_{1,1}= & -\frac{\underline{c}_{f}+\underline{c}_{r}}{m \bar{v}_{C o G}}=-23.6502, \\
\underline{a}_{1,2}= & \frac{\underline{c}_{r} \underline{l}_{r}-\bar{c}_{f} \bar{l}_{f}}{m \bar{v}_{C o G}^{2}}-1=-0.9811, \\
\bar{a}_{1,2}= & \frac{\bar{c}_{r} \bar{l}_{r}-\underline{c}_{f} \underline{l}_{f}}{m \underline{v}_{C o G}^{2}}-1=20.0328, \\
\underline{a}_{2,1}= & \frac{\underline{c}_{r} \underline{l}_{r}-\bar{c}_{f} \bar{l}_{f}}{J}-2 k \bar{h} l \frac{\bar{c}_{f}+\bar{c}_{r}}{b g \bar{l}_{f}}=2.86-55933 k, \\
\bar{a}_{2,1}= & \frac{\underline{c}_{r} \underline{l}_{r}-\underline{c}_{f} \underline{l}_{f}}{J}-2 k \underline{h l} \frac{\underline{c}_{f}+\underline{c}_{r}}{b g \underline{l}_{f}}=36.62-52008 k, \\
\bar{a}_{2,2}= & -\frac{\underline{c}_{r} \underline{l}_{r}^{2}+\underline{c}_{f} \underline{l}_{f}^{2}}{J \bar{v}_{C o G}}-2 k \bar{h} l \frac{\underline{l}_{f} \underline{c}_{f}-\underline{l}_{r} \underline{c}_{r}}{b g \underline{l}_{f} m \bar{v}_{C o G}} \\
\underline{a}_{2,2}= & -\frac{\bar{c}_{r} \bar{l}_{r}^{2}+\bar{c}_{f} \bar{l}_{f}^{2}}{J \underline{v}_{C o G}}-2 k \underline{h}_{l} \frac{\bar{l}_{f} \bar{c}_{f}-\bar{l}_{r} \bar{c}_{r}}{b g \bar{l}_{f} m \underline{v}_{C o G}} \\
= & -260.5-4.68 k, 0.871 k ; \\
= &
\end{aligned}
$$


With the above values 16 vertexes are built and solving Algorithm 1, with $k=0$ a $P$ matrix that satisfies $P A_{i}+A_{i}^{T} P \preceq 0$ is

$$
P=\left[\begin{array}{cc}
0.1338 & -0.0275 \\
-0.0275 & 0.0470
\end{array}\right] \times 10^{-10}
$$

For this iteration, $\gamma_{\max } \approx 0$. With $\Delta_{k}=0.02$, the next iteration results in $\gamma_{\max }=4.58 \times 10^{-6}$ and then $\gamma_{\max }=9.162 \times 10^{-6}$, successively such that the stability margin increases with $k$. In this case, any $k>0$ is enough to mitigate the RI, and it is tuned in such a range for to a desired response; that is, if the gain is selected high, a higher magnitude of perturbation can be rejected but it may seem aggressive for the driver (the rollover risk is lower), and if the gain is selected low, a lower magnitude of perturbation can be rejected (the rollover risk is higher); for the simulations presented in the following, $k=$ 9.63 is used.

In order to exhibit graphically the RI mitigation, consider the rollover manoeuvre of Figure 4 multiplied by 1.9 (190\%). Using this maneuver in open loop, as in Section 4.2, the left rear wheel of the tricycle loses contact with the ground and the vehicle ends its trajectory with an accident. In the upper plot of Figure 14 a comparative of the RI dynamic behaviour of the vehicle in OL (black plot) and several scenarios in CL (various colours) are shown. Such CL scenarios consist of relevant combinations of design limits of each uncertain parameter considered, including the friction coefficient. From this trend it can be noted that, for the different combinations of uncertain parameters in closed loop, the absolute value of the RI always remains lower than the unit while, in closed loop, values of $R I>1$ indicate a rollover; in this way, it can be seen that the controller is robust to changes in the parameters including the friction coefficient and manages to avoid the rollover of the vehicle compared to the scenario in open loop. The above affirmation can be pictorially confirmed in Figure 15; note that the black squared snap corresponding to the OL scenario shows how the vehicle performs until its rollover.

In the middle plot of Figure 14 the roll angle evolution in the aforementioned scenarios is shown. The black plot, corresponding to the OL scenario also confirms that the vehicle rolls upside down. In the bottom plot of Figure 14 the Yaw dynamics are graphed confirming the loss of vehicle manoeuvrability in OL and how the elusive trajectory is followed in CL.

From the previous results, it can be confirmed (see (27)) that high $\mathrm{CoG}$, low $l_{f}$, high $l_{r}$, and low $\mu$ are the worst scenario for rollover risk within the design boundaries (violet plots). It is worth mentioning that the tire stiffness $c_{f}$ and $c_{r}$ are timevarying and simulated by CarSim as nonlinear dynamics by the Pacejka approximation and the bounds for the robust controller design were selected from such approximation.

For purposes of completeness, in Figure 16 the normalized vertical forces in the rear wheels are plotted, as well as the RI for the worst scenario of parameter variation (within the design boundaries). Note that the vertical forces reach a zero value; although the rollover is mitigated, a greater perturbation or a parameter out of the design bounds can
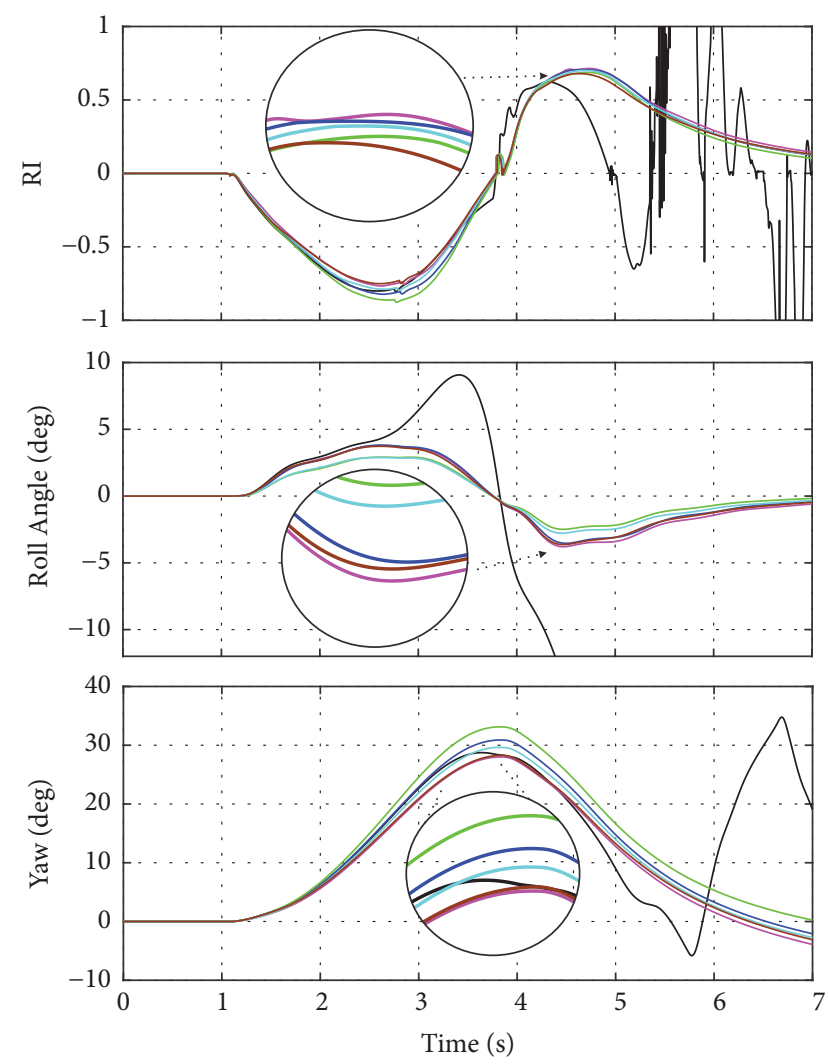

$$
\begin{aligned}
- \text { O.L., } \mu=0.85, \mathrm{~h}=0.49, \mathrm{l}_{\mathrm{f}}=1.12, \mathrm{l}_{\mathrm{r}}=0.99 \\
\text { C.L., } \mu=0.65, \mathrm{~h}=0.60, \mathrm{l}_{\mathrm{f}}=0.99, \mathrm{l}_{\mathrm{r}}=1.21 \\
\text { C.L., } \mu=0.65, \mathrm{~h}=0.60, \mathrm{l}_{\mathrm{f}}=1.21, \mathrm{l}_{\mathrm{r}}=0.99 \\
\text { C.L., } \mu=0.65, \mathrm{~h}=0.49, \mathrm{l}_{\mathrm{f}}=1.21, \mathrm{l}_{\mathrm{r}}=0.99 \\
\text { C.L., } \mu=0.65, \mathrm{~h}=0.49, \mathrm{l}_{\mathrm{f}}=0.99, \mathrm{l}_{\mathrm{r}}=1.21 \\
\text { C.L., } \mu=0.85, \mathrm{~h}=0.60, \mathrm{l}_{\mathrm{f}}=0.99, \mathrm{l}_{\mathrm{r}}=1.21
\end{aligned}
$$

FIGURE 14: Comparative of the RI relevant lateral dynamic behaviour of the vehicle in open loop (OL) and several CL scenarios with different parameters.

result in a rollover. That is, the presented strategy, as most of the active safety vehicular systems, is a driving assistance and at the moment it is physically impossible to avoid the rollover in every scenario. In Figure 17 the braking pressures for the worst scenario in CL are depicted.

In Figure 18 the RI, vertical forces in the rear wheels, and the roll angle for a scenario of parametric uncertainty out of the design bounds are shown. The parameter changed in this scenario is the vehicle speed set to $70 \mathrm{kmph}$. As mentioned previously, the controller presented in this paper can avoid the rollover as far as the limits of physics allow, but in extreme cases, as a very high speed, the differential braking is not enough to prevent the rollover.

Finally, the closed loop dynamical behaviour with an abrupt change of a parameter is illustrated; unfortunately not all of the parameters can be changed online in CarSim. In Figure 19 the RI, vertical forces in the rear wheels, and the roll angle for a scenario of an abrupt parameter change 


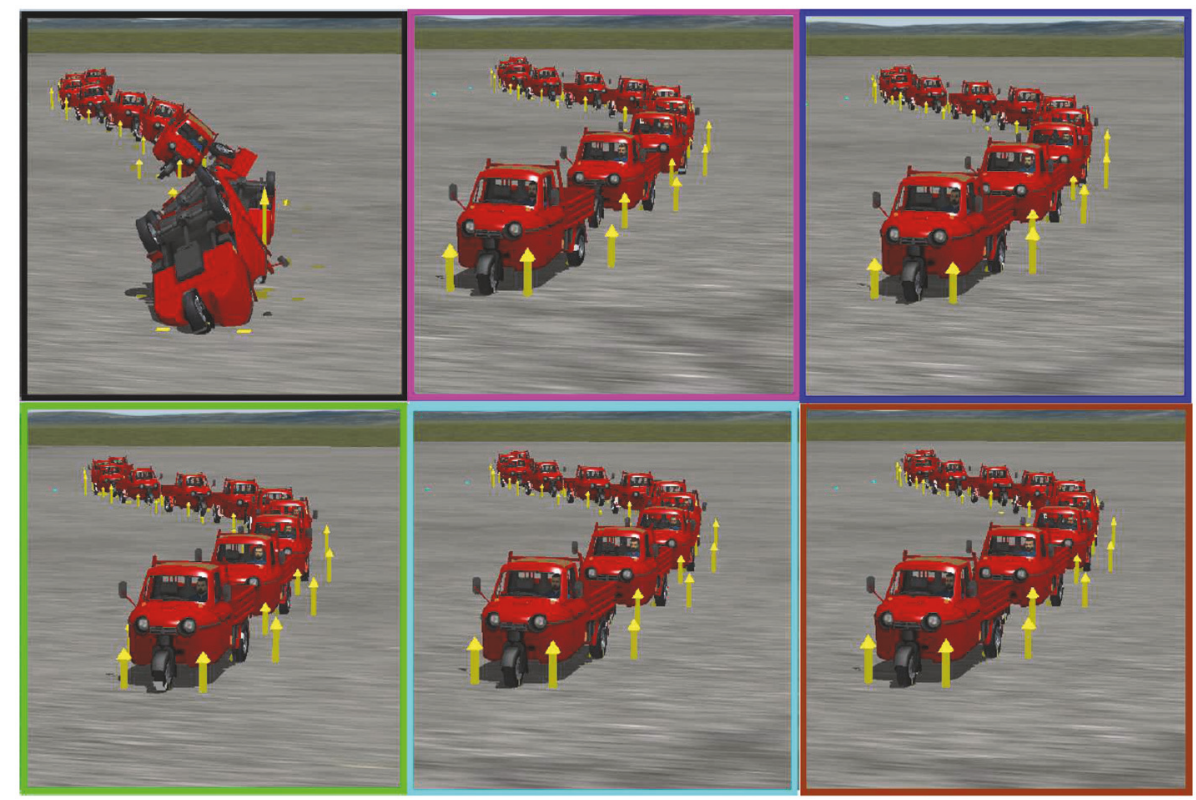

FIGURE 15: Comparative of the behaviour of the vehicle in OL and CL (Please see the colour of the square and use the legend of Figure 14 to determine the corresponding parameters.

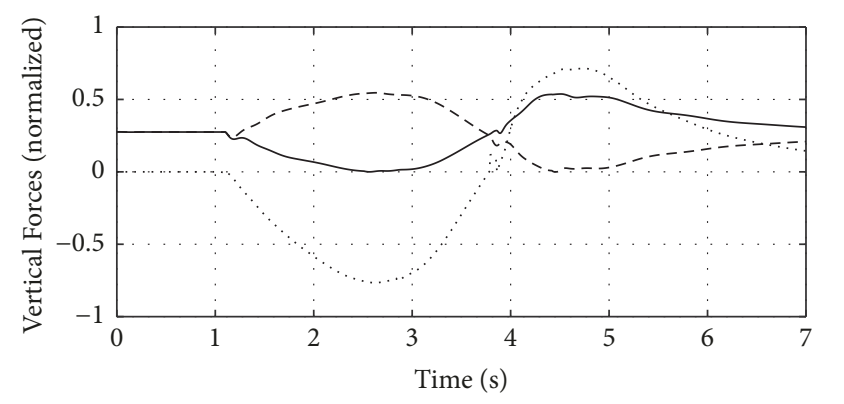

- Rear left vertical force
- - - Rear right vertical force
$\ldots$... RI

FIGURE 16: Tire-ground vertical forces for the worst scenario (within the design boundaries) in CL.

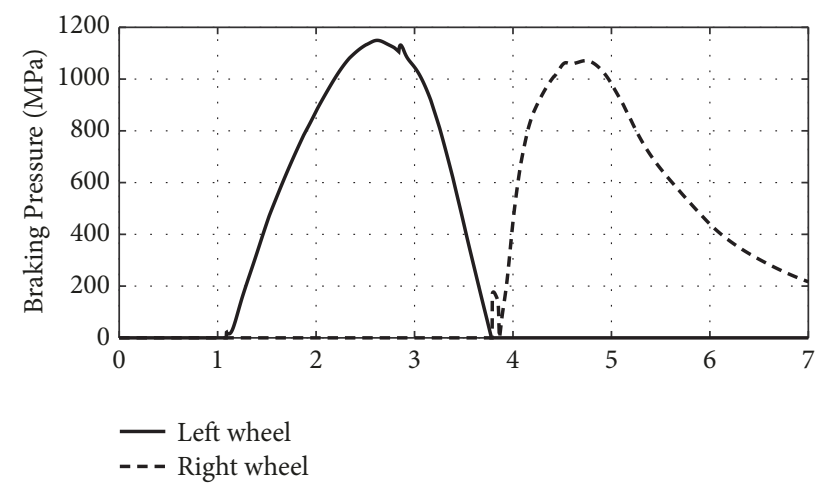

FIGURE 17: Controller braking pressures for the worst scenario (within the design boundaries) in CL. are shown: the CoG height is changed from 0.49 to $0.6 \mathrm{~m}$ in the worst scenario of parameter variation (within the design boundaries). This parameter, as well as the speed, is confirmed to be very sensitive to the rollover risk (by the previous simulations); however $h$ is selected to change abruptly because the tricycle speed cannot increase as fast as $h$ can. Note that even against such abrupt parameter change, the rollover is avoided.

\section{Final Discussion}

The presented analysis in this paper is aimed to analyse the lateral dynamics of an electric tricycle since its rollover is very hazardous for their occupants. First a mathematical model of the tricycle, useful for rollover detection, is obtained. Later, a dynamic rollover risk index is obtained from basic physic facts and the Newton Law. Such rollover index is useful to indicate the actual risk of rollover by a simple quantity. Then, a numerical validation of the mathematical model and the RI with very good results is performed. The RI can predict and detect a rollover condition, and one of their qualities is depending on the longitudinal and lateral position of the center of gravity, showing that even statically (in the sense of a straight line displacement) a rollover risk can exist. As far as the authors are aware, there is no such complete estimate of rollover risk in the literature.

Later, a rollover mitigation system is mathematically developed by using a polytopic description of the mathematical model. Such description allows designing an effective rollover mitigation system when the ranges of parametric variation are well estimated, and a Lyapunov type analysis allows ensuring the stability even against abrupt changes (values within compact ranges) in parameters. The effectiveness 


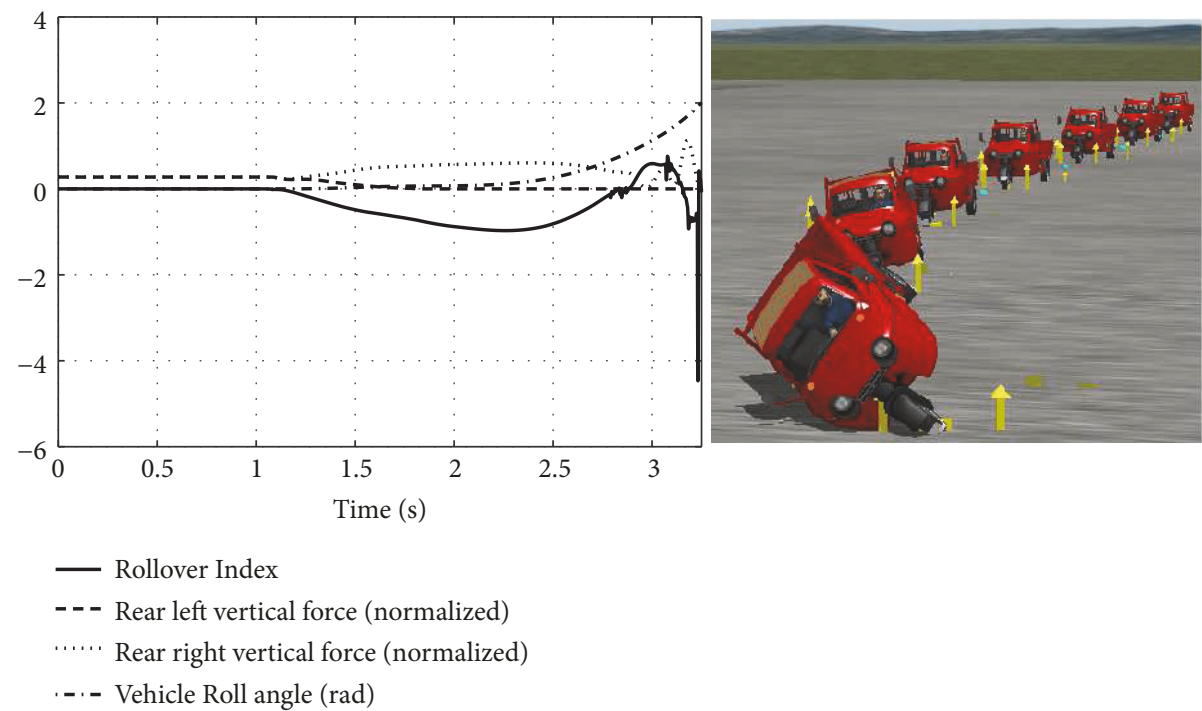

Figure 18: Vehicle dynamics for a closed loop scenario, out of the design boundaries.
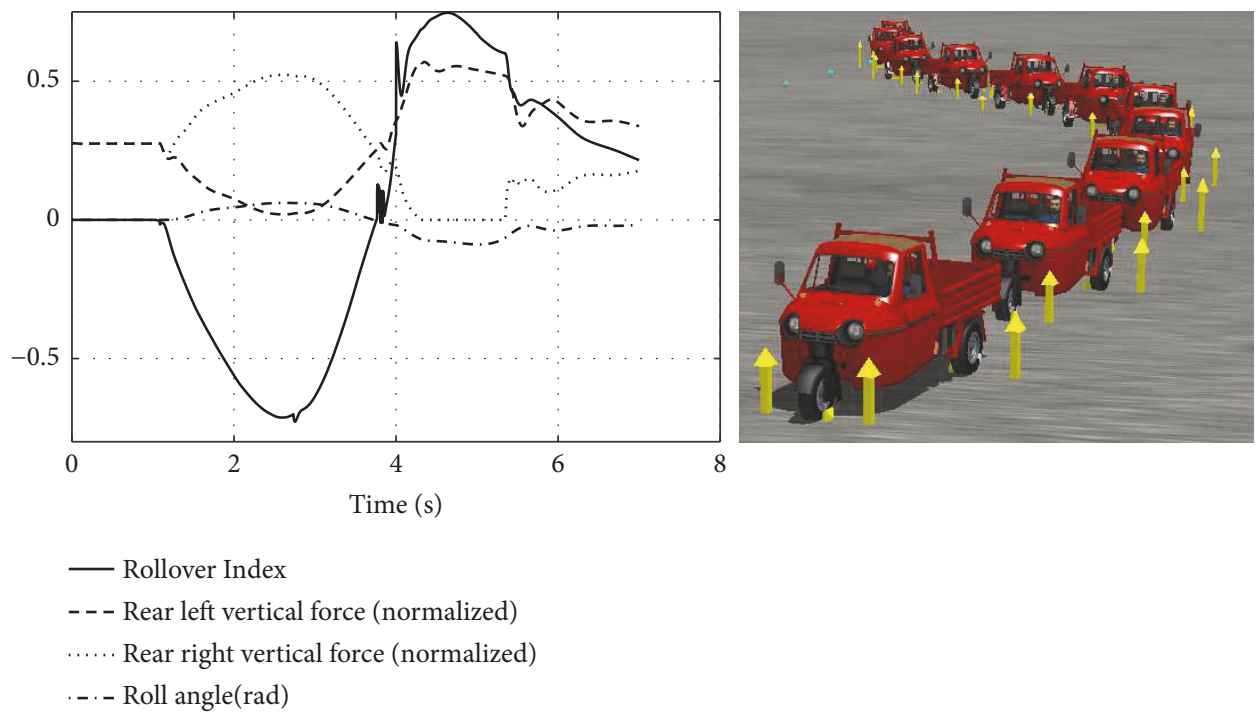

FIgURE 19: Vehicle dynamics for a closed loop scenario, with abrupt change of $h$ from 0.49 to 0.6 at second 4 .

of the controller is illustrated by using precise simulation software for a very representative rollover manoeuvre, showing that the controller is able to avoid the rollover.

It is worth mentioning that, from the previous simulations, a low friction coefficient has the effect of reducing the stability margin since the maximum breaking force is reduced. Even more, if the friction coefficient is too low a side slip occurs instead of a rollover; that is, there exists a relationship between the rollover and the sideslip during a curved maneuver depending on the friction coefficient, the CoG height, and other parameters as demonstrated in [32] for 4-wheeled vehicles. This last observation is out of the scope of this work and it is encouraged to the reader to ascertain it.

The implementation of a rollover mitigation device as presented in this paper can be implemented in a low cost platform conformed by low cost components: an accelerometer, a microcontroller, a power stage (with MOSFETS), and a pair of actuators. The actuators can be linear motors, electric pistons, or simple motors that wind each brake cable of the rear wheels (about 30 USD depending on the tricycle weight). More adequate and cheap actuators can be designed for a particular application; however, it is out of the scope of this paper. Since the controller law implies simple calculus (some mathematical operations, PWM, and sensor readings) a Microchip PIC/DSPIC can be used. The authors estimate an approximate total cost including a PCB fabrication on 100 USD.

\section{Conclusions}

In this work, a new estimation of the rollover risk in a tricycle during turns is presented. The estimation is presented/calculated as a simple quantity that even statically 
indicates the risk and its validation is performed with very good results.

In addition to showing that the use of robust controllers in polytopic systems has a wide range of applications, it is shown that a differential braking strategy, modelled as a polytopic system for the rear wheels of an electric tricycle, can mitigate the risk of rollover to where physical limitations allow it.

The controller is easy to implement since few components and a couple of actuators are required, which can be electrical or hydraulic.

\section{Nomenclature}

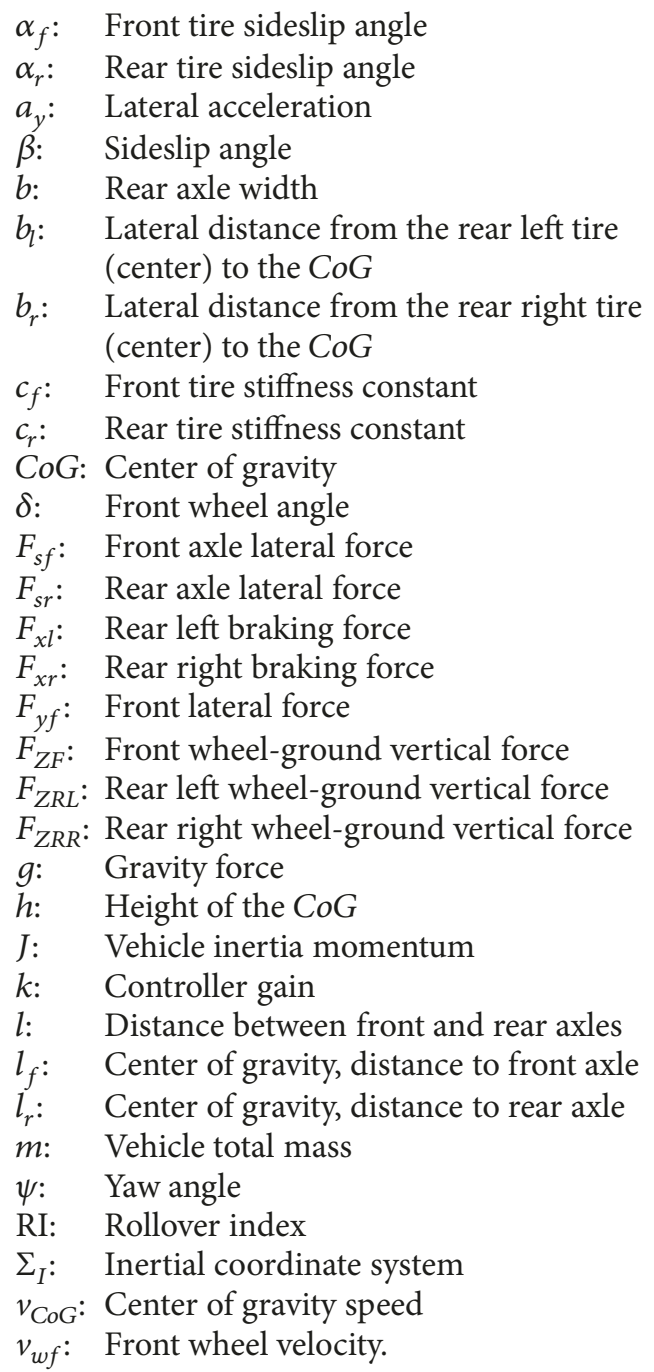

\section{Data Availability}

Datasets and CarSim model related to this article can be found at https://osf.io/ptyg4/?viewonly=e876fbf9d5394252b402080 bda3c902e, an open-source online data repository hosted at the Open Science Framework (Brian Nosek, 2018).

\section{Conflicts of Interest}

The authors declare no conflicts of interest for this paper.

\section{Acknowledgments}

The authors are grateful for the financial support of the National Council of Science and Technology, for the Cátedra 4155 and the scholarship of the third author.

\section{Supplementary Materials}

This paper was submitted including a highlight separate document; such document describes briefly the main paper contributions. (Supplementary Materials)

\section{References}

[1] A. Petroff, "These countries want to ban gas and diesel cars," CNN Money, 2017.

[2] W. Kehe and Y. Peng, "Research on Energy Substitution Terminal Energy Application Based on CRITIC Algorithm," in Proceedings of the 2017 10th International Conference on Intelligent Computation Technology and Automation (ICICTA), pp. 414-416, Changsha, October 2017.

[3] E. Barrett, B. Thayer, K. Studarus, and S. Pal, "The varied impacts of energy storage and photovoltaics on fossil fuel emissions," in Proceedings of the 2017 IEEE Power \& Energy Society General Meeting (PESGM), pp. 1-1, Chicago, IL, July 2017.

[4] S. Habib, M. M. Khan, F. Abbas, L. Sang, M. U. Shahid, and H. Tang, "A comprehensive study of implemented international standards, technical challenges, impacts and prospects for electric vehicles," IEEE Access, vol. 6, pp. 13866-13890, 2018.

[5] W. Li, X. Tan, B. Sun, and D. H. K. Tsang, "Optimal power dispatch of a centralised electric vehicle battery charging station with renewables," IET Communications, vol. 12, no. 5, pp. 579$585,2018$.

[6] W. J. Chang, K.-H. Lee, J.-I. Ha, and K. T. Nam, "Hydrogen production via water electrolysis: the benefits of a solar cellpowered process," IEEE Electrification Magazine, vol. 6, no. 1, pp. 19-25, 2018.

[7] J. Kim and S. Kim, "Obstacles to the success of fuel-cell electric vehicles: are they truly impossible to overcome?" IEEE Electrification Magazine, vol. 6, no. 1, pp. 48-54, 2018.

[8] B. M. Reddy, P. Samuel, and N. S. M. Reddy, "Government policies help promote clean transportation in india: protonexchange membrane fuel cells for vehicles," IEEE Electrification Magazine, vol. 6, no. 1, pp. 26-36, 2018.

[9] L. Li, Y. Lu, R. Wang, and J. Chen, "A three-dimensional dynamics control framework of vehicle lateral stability and rollover prevention via active braking with MPC," IEEE Transactions on Industrial Electronics, vol. 64, no. 4, pp. 3389-3401, 2017.

[10] J. J. Rath, M. Defoort, and K. C. Veluvolu, "Rollover index estimation in the presence of sensor faults, unknown inputs, and uncertainties," IEEE Transactions on Intelligent Transportation Systems, vol. 17, no. 10, pp. 2949-2959, 2016.

[11] H. Imine and M. Djemai, "Switched control for reducing impact of vertical forces on road and heavy-vehicle rollover avoidance," IEEE Transactions on Vehicular Technology, vol. 65, no. 6, pp. 4044-4052, 2016.

[12] N. C. Parida, S. Raha, and A. Ramani, "Rollover-preventive force synthesis at active suspensions in a vehicle performing a severe maneuver with wheels lifted off," IEEE Transactions on Intelligent Transportation Systems, vol. 15, no. 6, pp. 2583-2594, 2014. 
[13] M. Akar and A. D. Dere, "A switching rollover controller coupled with closed-loop adaptive vehicle parameter identification," IEEE Transactions on Intelligent Transportation Systems, vol. 15, no. 4, pp. 1579-1585, 2014.

[14] H. Imine, A. Benallegue, T. Madani, and S. Srairi, "Rollover risk prediction of heavy vehicle using high-order sliding-mode observer: experimental results," IEEE Transactions on Vehicular Technology, vol. 63, no. 6, pp. 2533-2543, 2014.

[15] A. Pandey, S. Jha, and D. Chakravarty, "Modeling and Control of an Autonomous Three Wheeled Mobile Robot with Front Steer," in Proceedings of the 2017 First IEEE International Conference on Robotic Computing (IRC), pp. 136-142, Taichung, Taiwan, April 2017.

[16] J. P. A. Ramoso and M. C. Ramos, "Comparative study of different Fuzzy-Neural configurations for autonomous vehicle following algorithm," in Proceedings of the 6th IEEE International Conference on Control System, Computing and Engineering, ICCSCE 2016, pp. 413-418, Malaysia, November 2016.

[17] D. Lopez de Luise, W. Bel, D. Mansilla, A. Lobatos, L. Blanc, and R. M. la Rosa, "Predicción de Riesgo basado en tiempo y patrones GPS," in Proceedings of the 2016 IEEE Biennial Congress of Argentina (ARGENCON), pp. 1-7, Buenos Aires, Argentina, June 2016.

[18] D. S. Vincent, S. Karthikeyan, and E. Manokaran, "Prevention of Accidents in Public Transport Corporation at Madurai City," in Proceedings of the 2011 International Conference on Management and Service Science (MASS 2011), pp. 1-5, Wuhan, China, August 2011.

[19] E. Austin, A. S. Christopher, O. Peter, E. W. Saturday et al., "Determination of center of gravity and dynamic stability evaluation of a cargo-type tricycle," American Journal of Mechanical Engineering, vol. 3, no. 1, pp. 26-31, 2015.

[20] J. H. Woodrooffe, Review of Canadian Experience with the Regulation of Large Commercial Motor Vehicles, vol. 671, Transportation Research Board, 2010.

[21] C. E. Beal and J. C. Gerdes, "Model predictive control for vehicle stabilization at the limits of handling," IEEE Transactions on Control Systems Technology, vol. 21, no. 4, pp. 1258-1269, 2013.

[22] D. Bernardiniy, S. Di Cairanoz, A. Bemporad, and H. Tsengz, "Drive-by-wire vehicle stabilization and yaw regulation: a hybrid Model Predictive Control design," in Proceedings of the 2009 Joint 48th IEEE Conference on Decision and Control (CDC) and 28th Chinese Control Conference (CCC), pp. 7621-7626, Shanghai, China, December 2009.

[23] M. A. R. Licea and I. Cervantes, "Robust switched predictive braking control for rollover prevention in wheeled vehicles," Mathematical Problems in Engineering, vol. 2014, Article ID 356250, 12 pages, 2014.

[24] H. Dahmani, O. Pages, A. El Hajjaji, and N. Daraoui, "Observerbased robust control of vehicle dynamics for rollover mitigation in critical situations," IEEE Transactions on Intelligent Transportation Systems, vol. 15, no. 1, pp. 274-284, 2014.

[25] P. Falcone, F. Borrelli, J. Asgari, H. E. Tseng, and D. Hrovat, "Predictive active steering control for autonomous vehicle systems," IEEE Transactions on Control Systems Technology, vol. 15, no. 3, pp. 566-580, 2007.

[26] U. Kiencke and L. Nielsen, Automotive Control Systems: for Engine, Driveline, and Vehicle, IOP Publishing, 2000.

[27] H. Pacejka, Tire and Vehicle Dynamics, Elsevier, 2005.

[28] J. Pearson, "Vehicle weights and dimension limits within the nafta partnership," Task Force on VWD Policy, vol. 27, 2002.
[29] S. Solmaz, "Switched stable control design methodology applied to vehicle rollover prevention based on switched suspension settings," IET Control Theory \& Applications, vol. 5, no. 9, pp. 1104-1112, 2011.

[30] S. R. Lay, "Convex sets and their applications," in Pure and Applied Mathematics, John Wiley and Sons, 1982.

[31] S. Boyd, L. El Ghaoui, E . Feron, and V. Balakrishnan, Linear Matrix Inequalities in System and Control Theory, SIAM, Philadelphia, Pa, USA, 1994.

[32] M. R. Licea and I. Cervantes, "Robust indirect-defined envelope control for rollover and lateral skid prevention," Control Engineering Practice, vol. 61, pp. 149-162, 2017. 


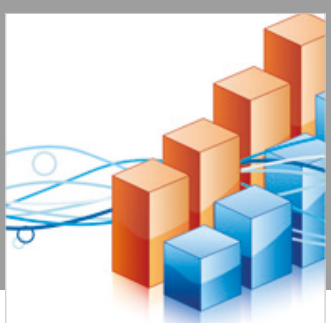

Advances in

Operations Research

\section{-n-m}
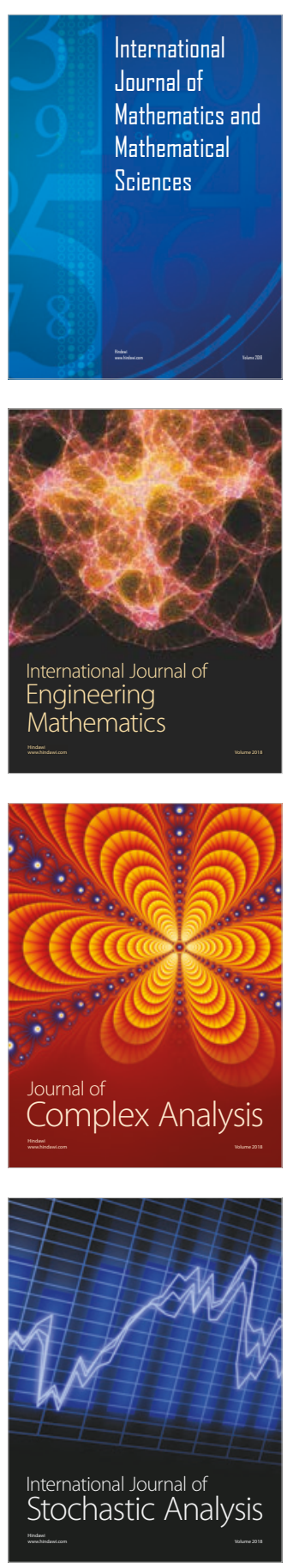
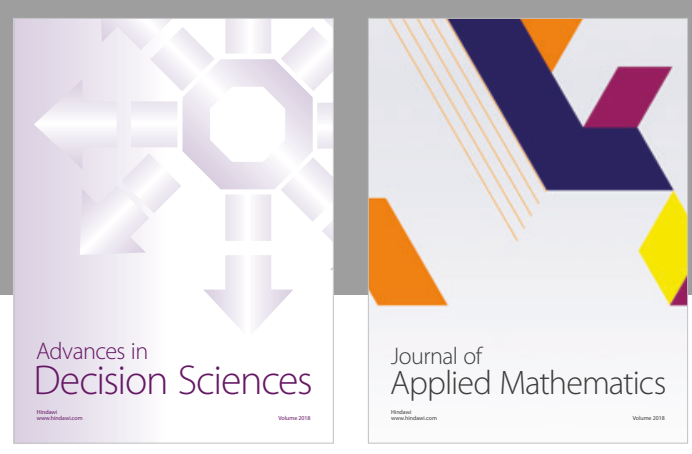

Journal of

Applied Mathematics
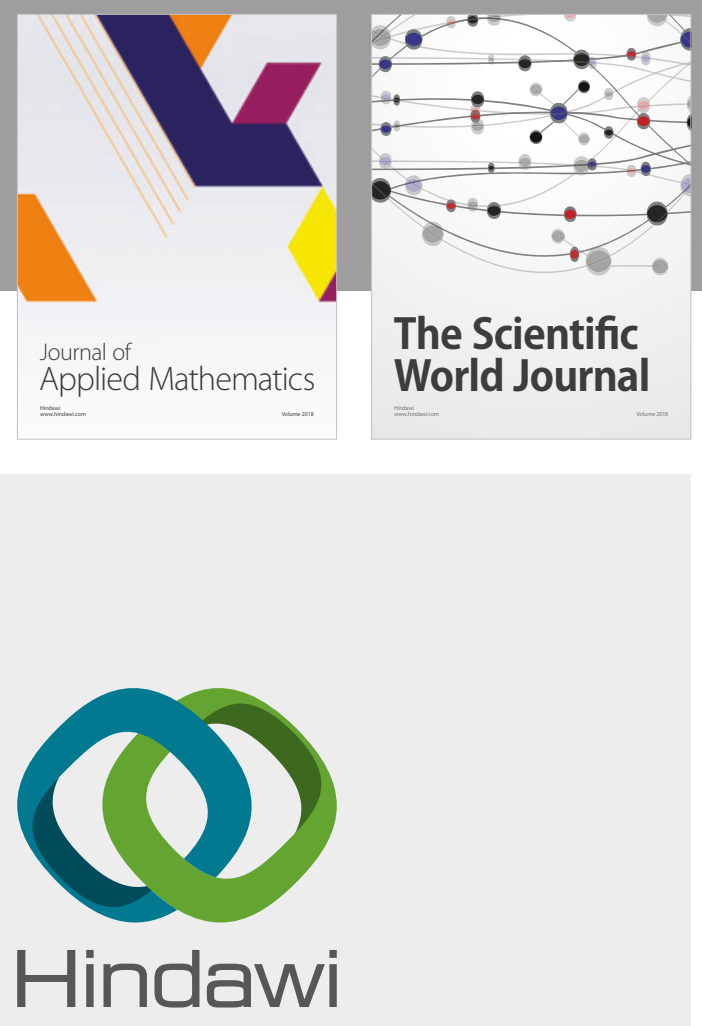

Submit your manuscripts at

www.hindawi.com

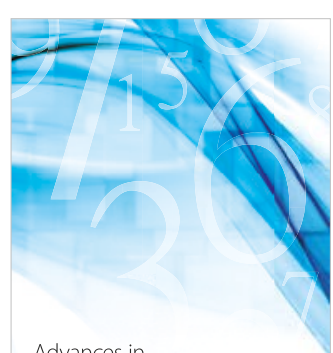

Advances in
Numerical Analysis
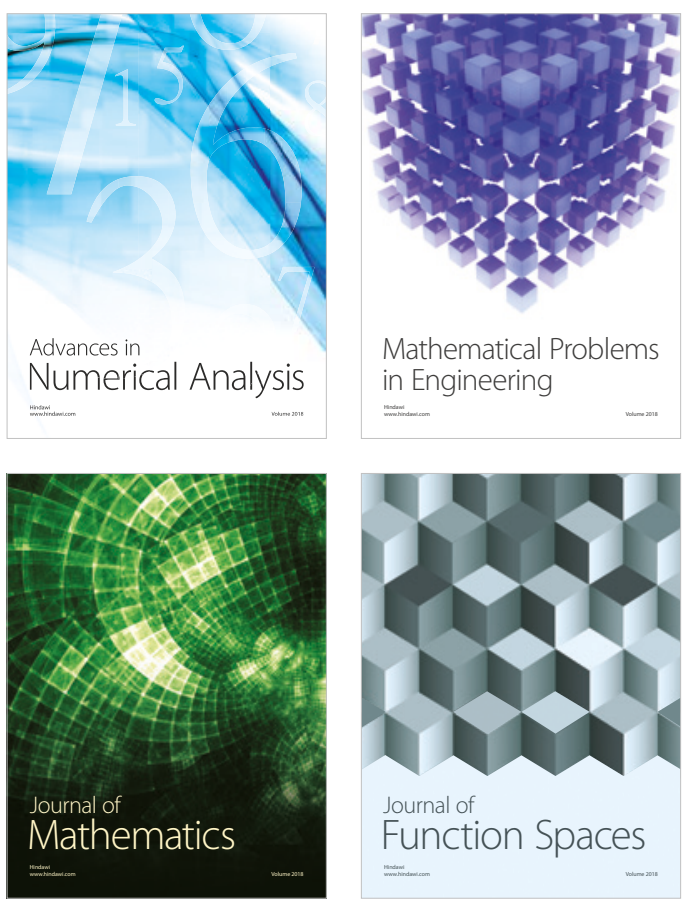

Mathematical Problems in Engineering

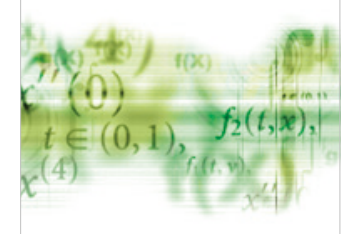

International Journal of

Differential Equations

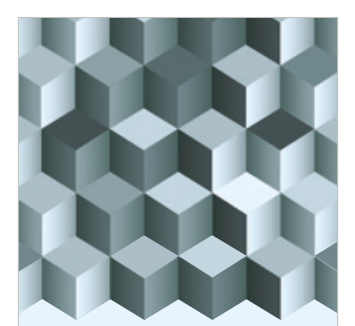

Journal of

Function Spaces
The Scientific

World Journal

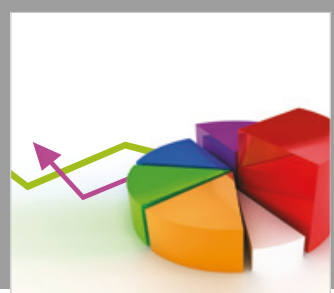

Journal of

Probability and Statistics
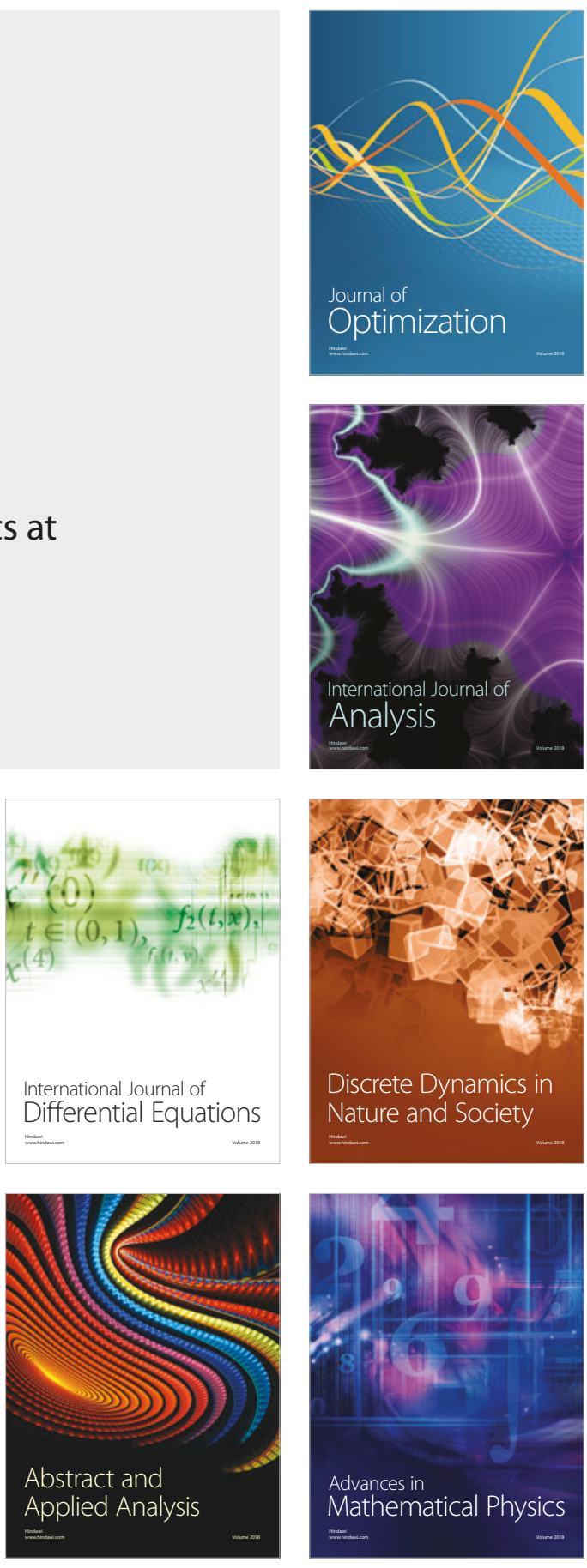